\title{
Cluster survey of the mid-altitude cusp - Part 2: Large-scale morphology
}

\author{
F. Pitout ${ }^{1}$, C. P. Escoubet ${ }^{2}$, B. Klecker ${ }^{3}$, and I. Dandouras ${ }^{4}$ \\ ${ }^{1}$ Laboratoire de Planétologie de Grenoble, CNRS/UJF, 122 rue de la Piscine, BP 53, 38041 Grenoble Cedex 9, France \\ ${ }^{2}$ European Space Agency, Keplerlaan 1, 2201 AZ, Noordwijk, The Netherlands \\ ${ }^{3}$ Max-Planck Institute für extraterrestrische Physik, Giessenbachstrasse, 85741 Garching, Germany \\ ${ }^{4}$ Centre d'étude Spatiale des Rayonnements, CNRS/UPS, 9 avenue du Colonel Roche, BP 4346, \\ 31028 Toulouse Cedex 4, France
}

Received: 6 May 2008 - Revised: 16 February 2009 - Accepted: 18 March 2009 - Published: 4 May 2009

\begin{abstract}
In this second part of our statistical study of the mid-altitude cusp, we compare the cusp morphology, as seen in the Cluster ion spectrometer (CIS), to the interplanetary magnetic field (IMF) orientation. We first recall the method we have used a) to define the cusp properties, b) to sort IMF conditions or behaviour in classes, c) to determine the proper time delay between the solar wind monitors and Cluster. Then, we define a few morphological features of the cusp and we relate these to the prevailing IMF. Our results reveal, among other things, that the occurrence of clearly dispersed ion structures in the cusp is $48 \%$. From these dispersions, we infer the distance to reconnection site, which we relate to external conditions. In all other cases, the cusp exhibits a more disturbed behaviour in terms of ion structures and fall in our "discontinuous" or "irregular" categories. Among these, a few interesting cases of discontinuous cusps occurring under stable IMF conditions have been identified. They all occur when the IMF is dominated by its Y-component, which plays in principle in favour of anti-parallel reconnection but their wide MLT and latitudinal distributions is a priori incompatible with the anti-parallel reconnection hypothesis solely.
\end{abstract}

Keywords. Magnetospheric physics (Magnetopause, cusp, and boundary layers; Magnetospheric configuration and dynamics; Solar wind-magnetosphere interactions)

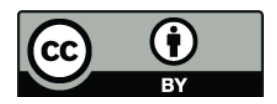

Correspondence to: F. Pitout (frederic.pitout@obs.ujf-grenoble.fr)

\section{Introduction}

Polar cusps are those regions of the magnetosphere where the solar wind has direct access to the Earth's environment. One may consider them as a monitor of the solar windmagnetosphere coupling. Their location and dynamics gives good indications on the connections between the interplanetary magnetic field (IMF) and the solar wind on the one hand, and Earth's magnetic field and environment on the other (Pitout et al., 2006b, referred to hereafter as paper 1). In this paper, we aim to link the morphology of the cusp to the state of coupling, and to relate our findings to the magnetic reconnection paradigm.

\subsection{Cusp precipitation and velocity filter effect}

There are many evidences showing that the cusp is on newly opened field lines and that cusp particles have the same properties as those of the solar wind. Indeed the very first insitu observations of the cusp showed that it is populated by heated magnetosheath particles (Heikkila and Winningham, 1971; Franck, 1971). The main physical process invoked to explain the penetration of shocked solar wind plasma in the Earth's magnetosphere is magnetic reconnection.

A consequence of the reconnection process is the velocity filter effect (Rosenbauer et al, 1975; Reiff et al., 1977; Burch et al., 1982). Magnetosheath ions are injected at the reconnection site and precipitate along the cusp open field lines. Those field lines move with the convection and the particles flowing along them undergo a time of flight effect that is inversely proportional to their energy (the higher the energy, the fastest the particle), resulting in energy dispersions. The dispersion is controlled by the direction and magnitude of the

Published by Copernicus Publications on behalf of the European Geosciences Union. 
convection and therefore, by the IMF orientation. Indeed, there are clear cause-effect relationships between the orientation of the IMF and the dispersion observed in the cusp. For southward IMF, as a newly opened field line moves poleward/tailward, the ion energy falls with increasing latitude (Reiff et al., 1977). For northward IMF on the other hand, the convection is directed towards the sun in the cusp region. The cusp ion population exhibits then a reversed dispersion, with energies increasing with latitude (Bosqued et al., 1985). For a detailed view on cusp precipitation and dynamics, the reader might want to read the review by Smith and Lockwood (1996).

Those cases could be tagged as text-book cases but in fact, the cusp exhibits a variety of large-scale morphologies (from nicely dispersed to highly disturbed) and of smaller scale features.

\subsection{Cusp morphology in ion data}

The footprints of dayside high-latitude magnetospheric regions have been extensively studied by Newell and coworkers using DMSP particle data (Newell and Meng, 1988; Newell et al., 1991a, b; Newell and Meng, 1992). These studies lead to a rigorous classification of each region and their corresponding sources: cusp, mantle, low-latitude boundary layer, etc. (Newell and Meng, 1988). From the morphology point of view, Yamauchi and Lundin (1994) analyzed Viking data and came up with a classification of the large- and meso-scale patterns of the energy-time dispersions observed in the cusp. Several large-scale patterns were identified:

- The southward IMF pattern with its characteristic dispersion: energies decreasing with increasing latitudes.

- The northward IMF pattern, which exhibit a reverse ion dispersion

- The three other classes gather the transient or unclear cusps (called "weak IMF" type and stagnant cusps)

The large-scale patterns are considered as spatial dispersion and therefore, they are supposed to give a good indication on the direction of the IMF. We shall investigate whether this is always true.

At smaller scale, i.e. within the main or large-scale dispersions, features are observed: stair-case ion structures or "multiple entries" (e.g. Woch and Lundin, 1991; Escoubet et al., 1992). It has always been a problem to determine whether those smaller-scale ion structures observed by midaltitude satellites in the polar cusp were of temporal or spatial origin. The problem is not only an observational issue; it has also great consequences on the physics of solar windmagnetosphere coupling. There have been pieces of evidence that such features (staircase ion signature) were the manifestation of pulsed reconnection at the magnetopause (Lockwood et al., 1992; Escoubet et al., 1992). Yet, in some occa- sions, those features were not temporal but spatial and stable (Trattner et al., 1999, 2002a, b). Let us note that these two approaches are not necessarily incompatible (Němeček et al., 2004). One the one hand, it has never been doubted that pulsed reconnection and IMF/SW pressure changes could create such features. On the other hand, it has been reported that reconnection can take place at two different locations at the magnetopause, in the same hemisphere or not, and generate spatial structures (Trattner et al., 2005).

\subsection{Cusp for a $B_{y}$-dominated IMF: evidence for double cusps}

Even if the Z-component of the IMF does play a key role in the reconnection process, the Y-component should not be neglected. In fact, the Y-component, apart from being responsible for the zonal dynamics of the cusp, plays an important role in the reconnection process itself especially when the Zcomponent is weaker (than the Y-component). A strong IMF $B_{y}$ is thought, within the frame of anti-parallel reconnection, to allow reconnection at the far dusk or dawn sides of the dayside magnetopause even under northward IMF, i.e. when the possible reconnection site is confined to a small area. Moreover, the idea that reconnection can take place at both lowand high-latitude simultaneously was put forward by several studies, interestingly based on different instruments.

Considering the classification of auroral forms by Sandholt et al. (1998a), the so-called type 1-aurora is associated with subsolar reconnection. Type 1-auroras are observed at typical cusp latitude for southward IMF (around $75^{\circ} \mathrm{MLAT}$ ). On the other hand, type 2-auroras due to lobe merging are located at higher latitudes and are consequently observed typically for northward IMF. However, cases of simultaneous observations of type 1- and type 2-auroral forms have been reported (Sandholt et al., 1998b, 2001). These so-called auroral bifurcations have been observed by all-sky cameras, whose wide field of view makes it possible to observe the whole dayside aurora display and dynamics over a large region of the ionosphere. Those observations suggest that the reconnection process may take place in some particular circumstances simultaneously at the dayside magnetopause and in the magnetospheric lobe.

Also from the ground, incoherent scatter radar data revealed that equatorward and poleward convection (indicative of dayside and lobe reconnection, respectively) occurred along the same line of sight (radar pointing north) during a period of By-dominated IMF, suggesting that reconnection took place at low- and high-latitudes simultaneously (Pitout et al., 2002).

Likewise, satellite particle data showed ion distribution functions consistent with ion being injected equatorward of the cusp for Northward IMF (Chandler et al., 1999; Fuselier et al., 2000; Russell et al., 2000). When the solar wind dynamic pressure is high, it is thought that interplanetary magnetic field lines and Earth's magnetosphere magnetic field 
lines may merge even without strict antiparallel conditions. More recently, Pu et al. (2005) using Double Star data also showed that component reconnection could operate at the low-latitude magnetopause under northward IMF.

Wing et al. $(2001,2004)$ have reported observations of double cusp crossings by DMSP satellites and have proposed a model explaining those observations in term of low- and high latitude merging occurring simultaneously. The model predicts the existence of two cusps so to speak, one at high latitude and another at lower latitude for a weak negative Zcomponent of the IMF and a strong Y-component. Within the higher latitude cusp, originating from the high-latitude magnetosheath, the $\boldsymbol{E} \times \boldsymbol{B}$ drift is thought to be strongly azimuthal and poleward, resulting in ion dispersion that looks like a typical southward IMF dispersion. On the other hand, the lower latitude cusp is thought to be located in a region of weak azimuthal $\boldsymbol{E} \times \boldsymbol{B}$ drift. The lower latitude cusp appears therefore dispersionless.

More recently, Pitout et al. (2006a) and Escoubet et al. (2007) have studied cases of cusp crossings by the Cluster fleet. Both studied showed that two sources of magnetosheath particles may be seen in the data following an abrupt rotation of the IMF. Consequently, we shall need to find out whether double cusps only result from changes in the IMF or whether, as Wing et al. (2001) suggested, they also occur under steady IMF conditions.

\subsection{Double cusps and reconnection hypotheses}

Current discussions and on-going works are dealing with the nature of magnetic reconnection but many questions are still left unanswered. Do the IMF and the Earth's magnetic field have to be strictly anti-parallel to allow reconnection to occur (Crooker, 1979) or does component reconnection apply? (Gonzalez and Mozer, 1974; Sonnerup, 1974). Both seem to occur but can they operate simultaneously? If not, under what circumstances one is favoured over the other? The study of double cusps will certainly enable us to find replies because the two models, anti-parallel and component reconnection, predict different merging sites and therefore, different locations for double cusps.

As a first step, we aim in this paper at studying statically cusp crossings by looking at our data set and trying to a) find out how often discontinuous cusps occur and under what solar wind/IMF conditions, b) identify potential double cusps for future detailed study

\section{Method and statistical criteria}

In order to perform a statistical study of cusp crossings, we need four basic elements: criteria for orbit selection, a few criteria that will allow us to define and thus to identify the cusp, a few typical cusp morphologies that will allow us to classify the cusp crossings, and the IMF orientation corresponding to each crossing.

\subsection{Cusp location and orbit selection}

The orbits selected for participation in this statistical study are taken from twelve months. For each of the years 2001 through to 2004, we have studied 40 Cluster orbits from 1 July to 31 October. This period of each year was chosen because the Cluster spacecraft are then orbiting at middle altitude in the dayside magnetosphere between 16:00 and 08:00 MLT, respectively. As one of the goals of this study is the identification of double cusps, we have deliberately taken a wide MLT interval ( $\pm 4 \mathrm{~h}$ on both sides of magnetic noon) as reconnection sites leading to double cusps are expected to be located far away from 12:00 MLT (under strong IMF- $B_{y}$ ). Out the 960 possible cusp crossings (40 orbits/year times 4 years times 2 hemispheres times 3 spacecraft), only 261 actual cusp crossings were identified (paper 1).

\subsection{Cusp plasma properties}

The criteria that we have used to define the cusp are based on previous studies on cusp plasma at mid- or low-altitude (Newell and Meng, 1988). Our study is based on measurements performed by the CIS ion spectrometer (Rème et al., 2001) and the Flux Gate Magnetometer (Balogh et al., 2001) on board the Cluster spacecraft (Escoubet et al., 2001). The criteria are introduced in paper 1 . They can be summarized as follows:

- Density in the cusp equal or greater than that in the solar wind.

- Energy flux $F_{i}$ of precipitating cusp particles (a few $\mathrm{keV} / \mathrm{e}$ ) greater or equal than $10^{7} \mathrm{eV} / \mathrm{cm}^{2}-\mathrm{s}-\mathrm{sr}-\mathrm{eV}$.

- Energy flux $F_{i}$ lower than $10^{6} \mathrm{eV} / \mathrm{cm}^{2}$-s-sr-eV for energies above $10 \mathrm{keV} / \mathrm{e}$.

\subsection{Morphological classification}

The cusp at middle altitude indeed exhibits a great variety of morphologies. The cusp crossings were grouped into four classes (identified by visual inspection) as follows:

- Type 1: dispersed cusps. Those cusps exhibit the textbook velocity filter effect with high-energies at low latitudes and lower energies at high latitudes. Those cusps are expected to occur under southward IMF when the convection is anti-sunward. Figure 1 shows an example of dispersed cusp measured by the HIA sensor on board SC1 with, from top to bottom: an energy-time spectrogram, the ion density, and the three GSM components of the ion velocity perpendicular to the local magnetic field line (convection velocity). 
CIS-HIA RUMBA (SC 1) $10 / \mathrm{Sep} / 2002$
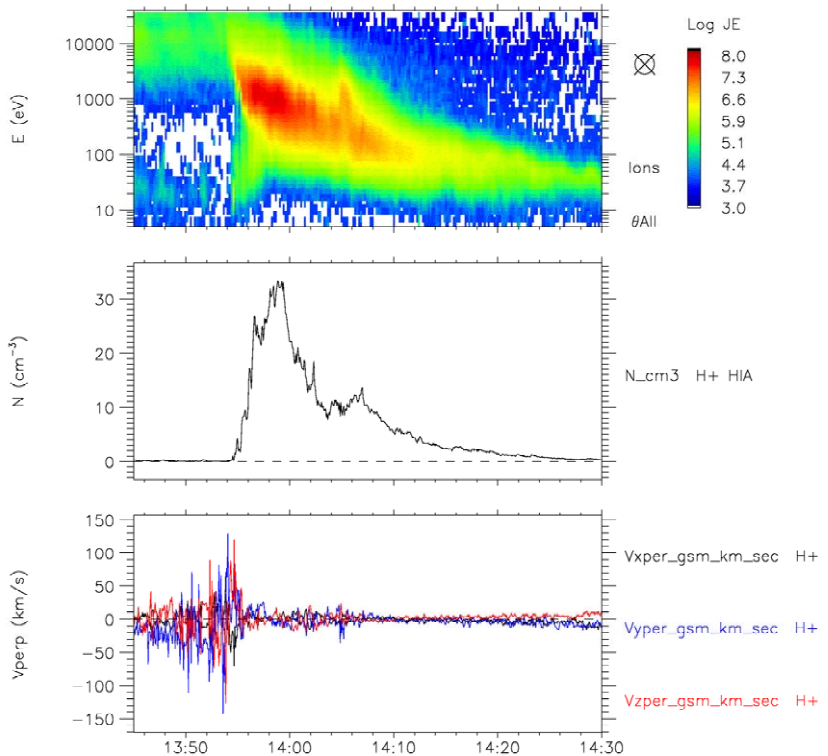

$\begin{array}{lccccc}\text { XGSE } & 3.90 & 3.68 & 3.44 & 3.16 & 2.90 \\ \text { YGSE } & -1.55 & -1.69 & -1.82 & -1.95 & -2.06 \\ \text { ZGSE } & 2.81 & 3.18 & 3.51 & 3.91 & 1.21 \\ \text { DIST } & 5.05 & 5.15 & 5.26 & 5.40 & 5.53\end{array}$

Fig. 1. Example of dispersed cusp under Southward IMF. From top to bottom: ion time-energy spectrogram, number density and convection velocity in GSM from Cluster-CIS.

- Type 2: reverse dispersed cusps. Those cusps show dispersions with high-energy ions at high latitudes and lower-energy ions at lower latitude. They are typically expected for northward IMF (lobe reconnection) when the convection is sunward in the cusp (Fig. 2).

- Type 3: discontinuous cusps. We define discontinuous cusps as having at least two components in the ion spectrogram, among which at least one clear large-scale dispersion (Fig. 3). This category will particularly draw our attention, as it is there that we expect cases of double cusps.

- Type 4: irregular cusps. We will consider a cusp crossing as irregular basically when it won't fit in any other categories. A priori, those cusps will be highly variable and structured. We can foresee that they will occur under highly variable IMF (Fig. 4).

- Determining the prevailing IMF.

In order to have the applicable IMF for each crossing, we have used the magnetic field instrument (MAG) and the solar wind plasma instrument (SWE) on board the ACE spacecraft. The propagation time from ACE to Cluster is first roughly estimated by dividing the solar wind bulk velocity by the
CIS-HIA SAMBA (SC 3) 08/Sep/2002
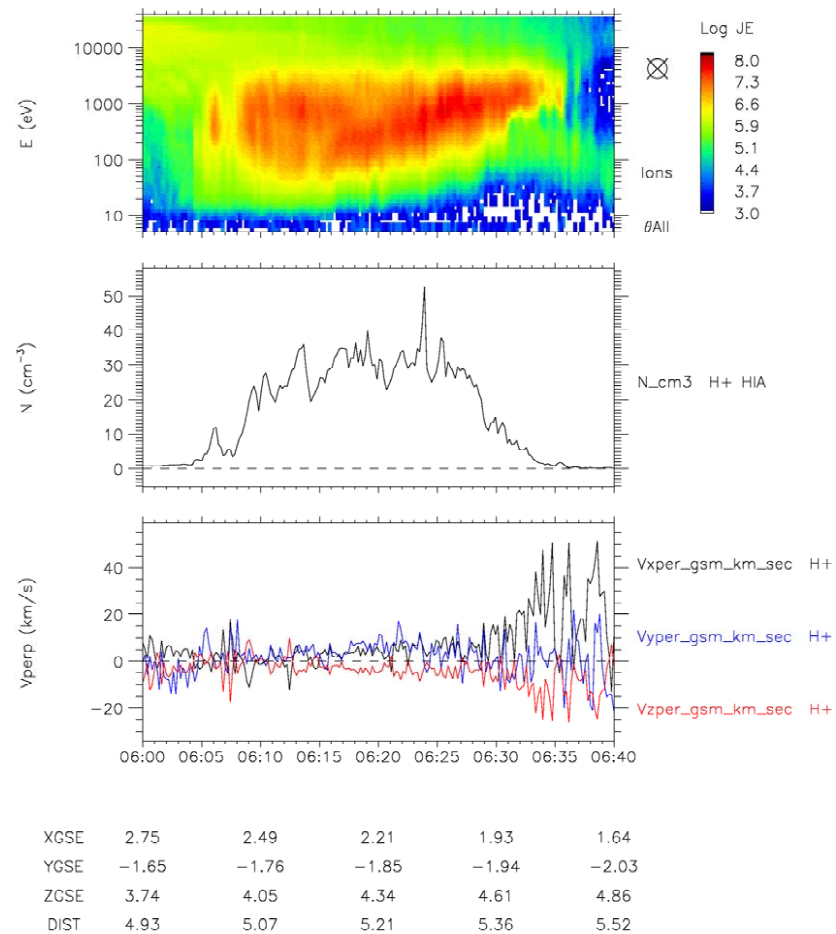

Fig. 2. Example of dispersed cusp under Northward IMF (reverse dispersion). From top to bottom: ion time-energy spectrogram, number density and convection velocity in GSM from Cluster-CIS.

distance between L1 and the dayside magnetosphere. When necessary, we check this lag by comparing ACE data to either Geotail data when suitably positioned in the near-Earth upstream solar wind or to ground instruments. Based on this, we sort any given cusp crossing among four classes of IMF behaviour:

a) Steady southward IMF during the whole cusp crossing.

b) Steady northward IMF during the whole cusp crossing.

c) Rotating IMF. This behaviour is chosen when one given change in the IMF orientation is clearly identified as occurring during the cusp crossing and as being responsible for a cusp discontinuity (presumably due to the motion of the latter).

d) Highly variable IMF. Some of the cusp crossings we found occur under very variable IMF to such an extent that we cannot isolate the IMF turning(s) responsible for the change(s) in cusp morphology

\section{Statistics of cusp morphologies}

Out of 261 cusp crossings, 100 exhibit a normal dispersion (Type 1), 25 a reversed dispersion (Type 2), 62 are classified as discontinuous (Type 3 ) and 74 as irregular (Type 4). 
CIS-CODIF TANGO (SC 4) 05/Sep/2002
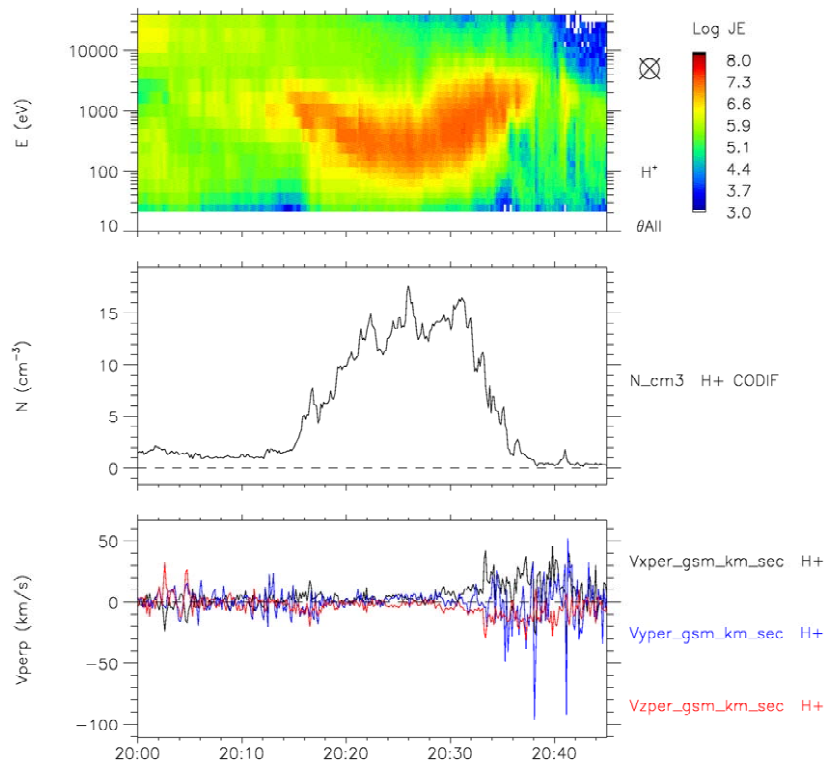

$\begin{array}{lccccc}\text { XGSE } & 3.12 & 2.86 & 2.58 & 2.26 & 1.96 \\ \text { YGSE } & -1.32 & -1.48 & -1.63 & -1.77 & -1.90 \\ \text { ZGSE } & 3.07 & 3.45 & 3.80 & 4.16 & 4.47 \\ \text { DIST } & 4.57 & 4.72 & 4.87 & 5.06 & 5.24\end{array}$

Fig. 3. Example of discontinuous cusp. From top to bottom: ion time-energy spectrogram, number density and convection velocity in GSM from Cluster-CIS

We have presented the results in Table 1, which shows for each type, the number of occurrence corresponding to each prevailing IMF conditions. We find the expected tendency: type 1-cusps occur mainly under southward IMF, type 2 under northward IMF, type 3 under rotating IMF, and type 4 under variable IMF. In this section, we shall analyze these results in more details.

\subsection{Occurrence of dispersed and reversed cusps}

It first appears that textbook cases of nicely dispersed ion structures in the cusp expected for steady IMF, either southward or northward, do no represent a majority of cases. We have found only 100 and 25 cases of them respectively out of 261 cusp crossings, which represent roughly $38 \%$ and $10 \%$ of all the crossings respectively. This mainly reflects the behaviour of the IMF. It means that more or less half of the time, the cusp was crossed while the IMF was steady. Indeed, most of them (85 out of 100 and 23 out of 25 ) occur during a steady IMF with the expected sign: negative for type 1- and positive for type 2-cusps (Table 1).

Figure 5 shows the distribution of these crossings. The upper plot displays the clock angle corresponding to the type 1and 2-cusps (in red and blue respectively). Dispersed cusps

$$
\text { CIS-HIA SAMBA (SC 3) 21/Aug/2003 }
$$
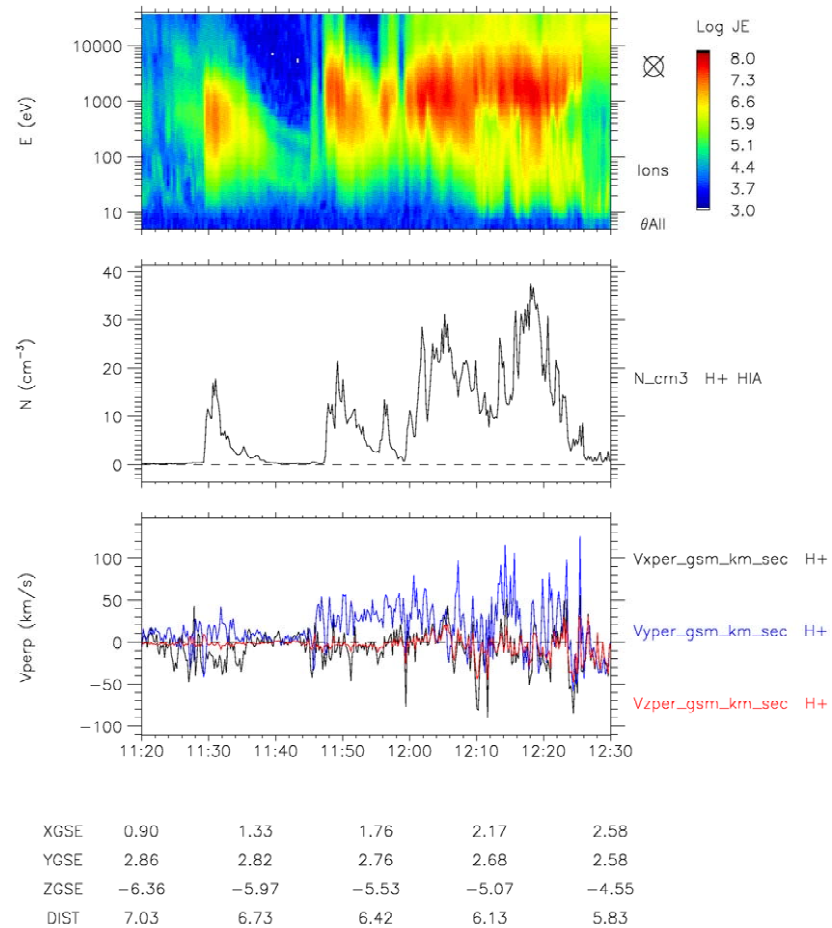

Fig. 4. Example of irregular cusp. From top to bottom: ion timeenergy spectrogram, number density and convection velocity in GSM from Cluster-CIS.

Table 1. Statistics of the cusp morphologies and their corresponding IMF conditions.

\begin{tabular}{cccccc}
\hline $\begin{array}{c}\text { Cusp } \\
\text { type }\end{array}$ & $\begin{array}{c}\text { All } \\
\text { IMF }\end{array}$ & $\begin{array}{c}\text { Steady } \\
\text { south }\end{array}$ & $\begin{array}{c}\text { Steady } \\
\text { north }\end{array}$ & Rotating & Variable \\
\hline 1 & 100 & 85 & 3 & 2 & 10 \\
2 & 25 & 0 & 23 & 1 & 1 \\
3 & 62 & 2 & 10 & 33 & 17 \\
4 & 74 & 14 & 8 & 4 & 48 \\
\hline
\end{tabular}

do occur under southward IMF while reversed cusps (type 2) occur under northward IMF. The lower plot shows the cusp crossings on a MLT-ILAT graph. Again, type 1- and type 2cusps are coloured in red and blue. The cusp is detected mainly in an area between $\sim 73^{\circ}$ and $\sim 806$ o ILAT and between 10:00 and 14:00 MLT. Thanks to the colours, we can verify by eye a well-known result (e.g. paper 1): under southward IMF, the cusp is located (or moves to) lower latitudes than under northward IMF.

Also, let us note that some cases of dispersed cusps occur under variable IMF. Indeed, Table 1 shows 2 and 10 type- 1 cusps were recorded while the IMF was rotating and variable 

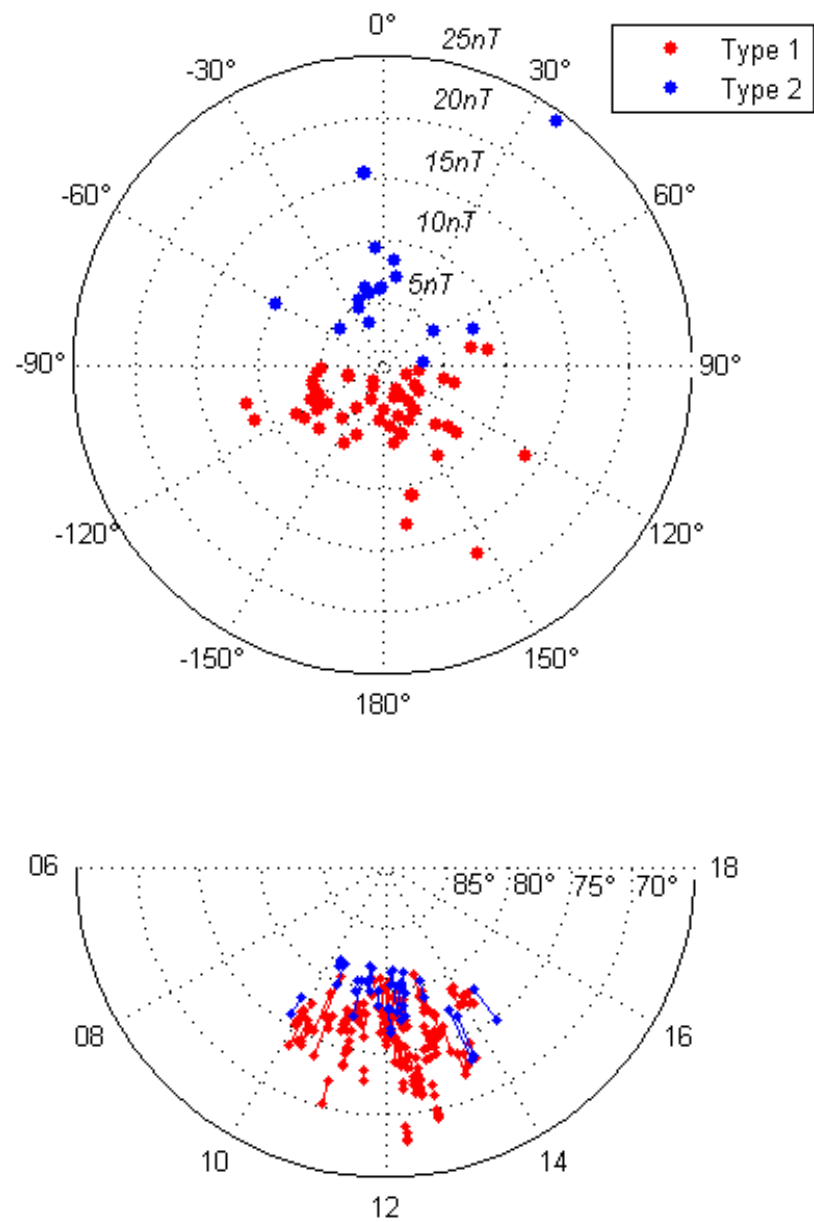

MLT

Fig. 5. IMF clock angles corresponding to type 1 and type 2 cusps (top) and their projections into a MLAT-ILAT polar plot (bottom).

respectively. Likewise, one type- 2 cusp occurred under rotating IMF and another occurred under variable IMF.

\subsection{Occurrence of discontinuous and irregular cusps}

Discontinuous and irregular cusps, as defined in Sect. 2.3, are detected 62 and 74 times, representing $24 \%$ and $28 \%$ of our samples, respectively.

Unsurprisingly, it appears that most of them correspond to changes in IMF orientation. These consequently result from a displacement of the cusp region and/or a change in the reconnection regime. Indeed, irregular and discontinuous cusps are, according to our results, mainly due to either an identified IMF turning or occur under highly variable IMF: 33 out of 62 type- 3 cusps correspond to a rotation of the IMF, while 48 out of 74 type- 4 cusps are detected when the IMF is variable.

Table 1 shows cases of discontinuous and irregular cusps (types 3 and 4) that occurred under stable IMF. Under stable
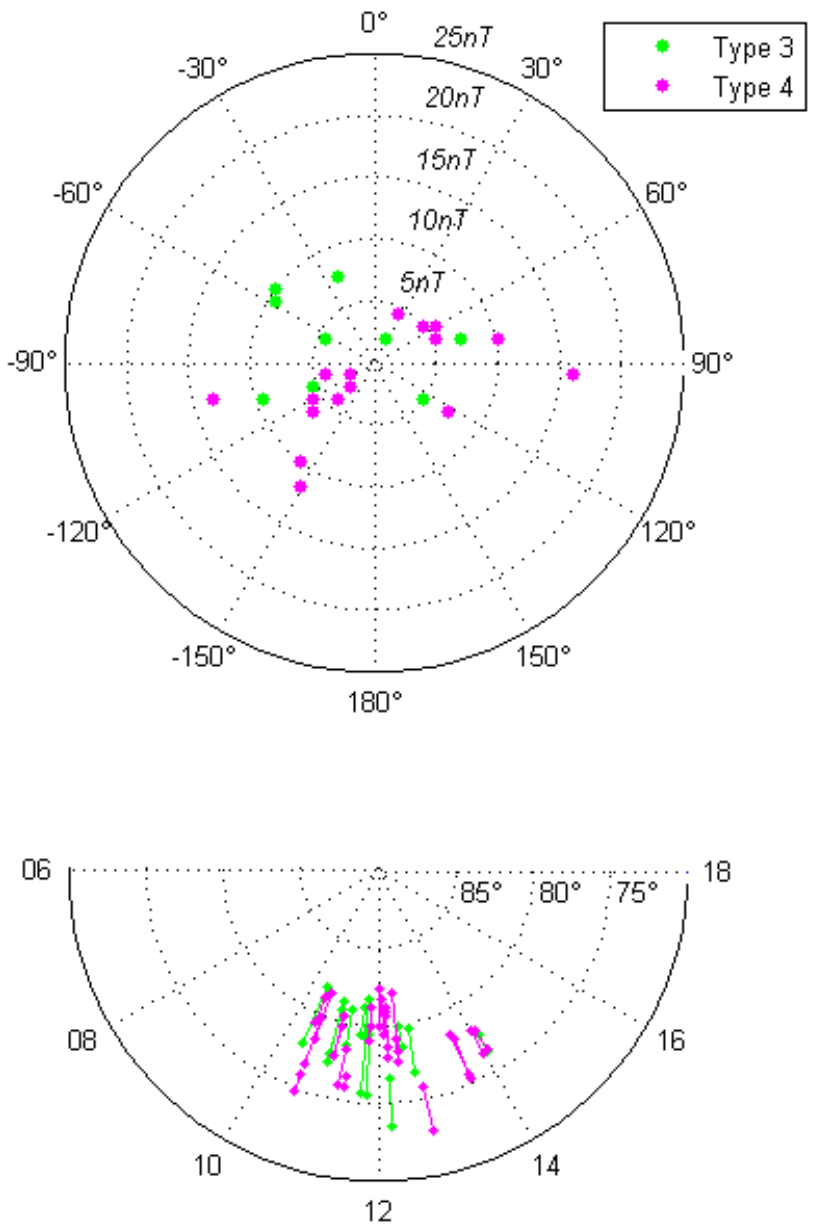

MLT

Fig. 6. Top panel shows the clock-angle of the IMF corresponding of the observed discontinuous and irregular cusps occurring under steady IMF. For those cusp crossings, the bottom panel shows the satellite path projected on a MLT-ILAT diagram.

IMF, we have 12 type- 3 cusps ( 2 under southward and 10 under northward IMF) and 22 type- 4 cusps ( 14 under southward and 8 under northward IMF). These cases are of particular interest for us, as they may be double cusps. We do not intend here to study them individually in details, we leave this for later. Meanwhile, we can study under which IMF conditions those discontinuous cusps occurred and where they are located.

The top panel of Fig. 6 shows the clock-angle of the IMF corresponding to the observed discontinuous and irregular cusps occurring under steady IMF. It appears clearly that all these cases occur under dominant or substantial IMF- $B_{y}$. As a matter of fact, there is one case that occurs under dominant and positive $\mathrm{B}_{z}$ (clock angle: $\sim-20^{\circ}$ ). This case is under investigation (Pitout et al., 2009). 
Apart from this latter case, all the other favour the antiparallel reconnection hypothesis that predicts the formation of double cusp under dominating $B_{y}$. However, things get somewhat less clear when one looks at the MLT at which those discontinuous cusps are observed (bottom panel of Fig. 6). As a matter of fact, they are not observed only early in the morning sector or late in the afternoon sector, but at all MLT.

A peculiar detail is that most of our cases correspond to relatively high solar wind density $\left(12 \mathrm{~cm}^{-3}\right.$ on average) whereas the corresponding dynamic pressure is not that high $(\sim 4 \mathrm{nPa}$ on average).

\subsection{Change of morphology following a rotation of the IMF}

In paper 1, we took advantage of the multipoint capability of the Cluster mission to study the reactivity of the cusp when the IMF rotates. We use the same principle here but to study qualitatively the changes of morphology that follow a rotation of the IMF.

Figure 7 displays changes in morphology following changes in the IMF. Each line shows the cusp type before the change of IMF and morphology (if any). For instance, to see the morphological evolution of type- 1 cusps, one has to look at the lowest horizontal line, labelled "Type 1". Then, each change is materialized by an arrow. Any given arrow has the following properties:

- Its origin indicates the cusp morphology (as explained above) and the value of $B_{z}$ (horizontal axis in $\mathrm{nT}$ ) before the change in the IMF.

- Its horizontal component indicates the sign of the $B_{z}$ variation: $B_{z}$ decreases if the arrow points towards the left and increases if the arrow point towards the right. The vertical component of the arrow indicates whether the cusp changes morphology: upwards if the cusp morphology changes to an upper type (type 1 to type 3 for instance) or downward if it changes to a lower type. A horizontal arrow means that the cusp has undergone no change in morphology.

- Its length is proportional to the variation in $B_{z}$.

- Its colour indicates the cusp type observed after the change in the IMF (same colour coding as labels of Yaxis).

The top panel of Fig. 7 shows changes between two spacecraft crossing the same cusp whereas the bottom panel shows changes between one spacecraft crossing the southern cusp and a spacecraft (it may be the same) crossing the northern cusp. Obviously, only cases where the IMF was measured (i.e. steady or rotating IMF conditions) were considered, which limit considerably the number of data points.
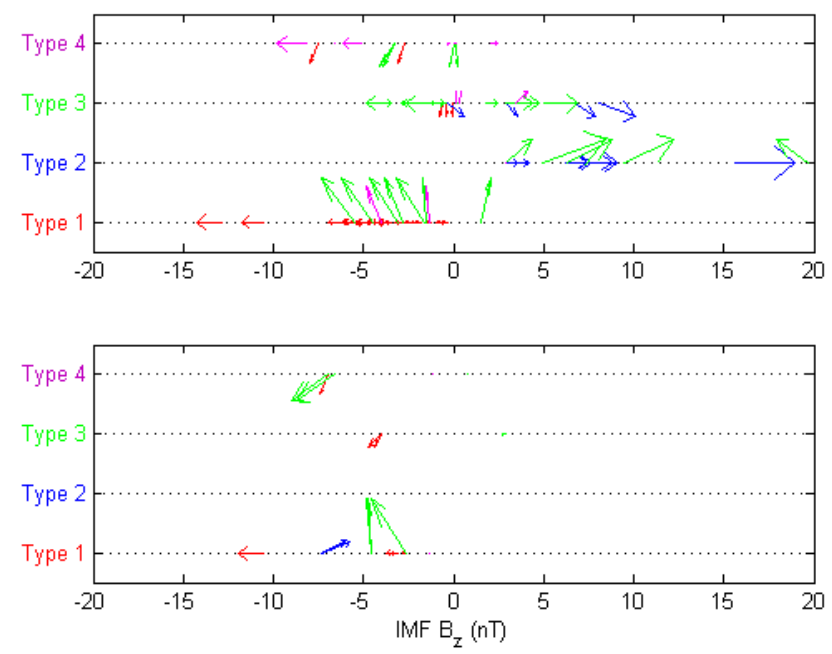

Fig. 7. Change in the cusp morphology following an IMF rotation. Each arrow has the following properties: (a) its origin shows the morphology of the cusp and the IMF- $B_{z}$ before the IMF change, (b) its color indicates the morphology of the cusp after the IMF change, (c) its direction point towards the morphological type after the IMF change, (d) its length is proportional to $\Delta B_{z}$. The top panel shows changes occurring during successive passes through the same cusp while the bottom panel shows successive passes through the southern and northern cusp (see explanations in paper 1).

In the top panel, we see that type 1- and type 2-cusps keep their morphology if the Z-component of the IMF keeps its sign. A type- 1 cusp remains a type- 1 if the $B_{z}$ remains or becomes more negative (horizontal red arrows on type-1 line) and type- 2 cusps remains type- 2 when $B_{z}$ remains positive (horizontal blue arrows on type-2 line). However, the cusp becomes discontinuous (type 3 ) or irregular (type 4) when the IMF- $\mathrm{B}_{z}$ changes sign: all non-horizontal arrows starting from type-1 and type- 2 lines are green or purple. In other words, the cusp does not pass directly from type- 1 to type- 2 (and vice versa) within the duration separating two satellites passes (no blue arrows starting from the "type 1" line nor red arrows starting from the "Type 2" line). This means that like its width (paper 1), the cusp cannot fully reorganize itself on large scales within $\sim 15$ min following an IMF turning, although we do know that the cusp reacts locally very fast to a change in the IMF (Pitout et al., 2006a; Escoubet et al., 2007). This also suggests that a discontinuous or irregular cusp, possibly with a transient dual source of magnetosheath plasma (Pitout et al., 2006a) is a necessary intermediate step. Indeed, there are red and blue arrows starting from the type 3 and type 4 lines. This indicates that from a discontinuous or irregular morphology, the cusp may become a type 1 if the IMF remains or turns southward (red arrows towards the left) or a type 2 if the IMF remains or turns northward (blue arrows towards the left).

We do not obtain much information from the bottom panel but still, we see one case of type 1-cusp becoming directly a 

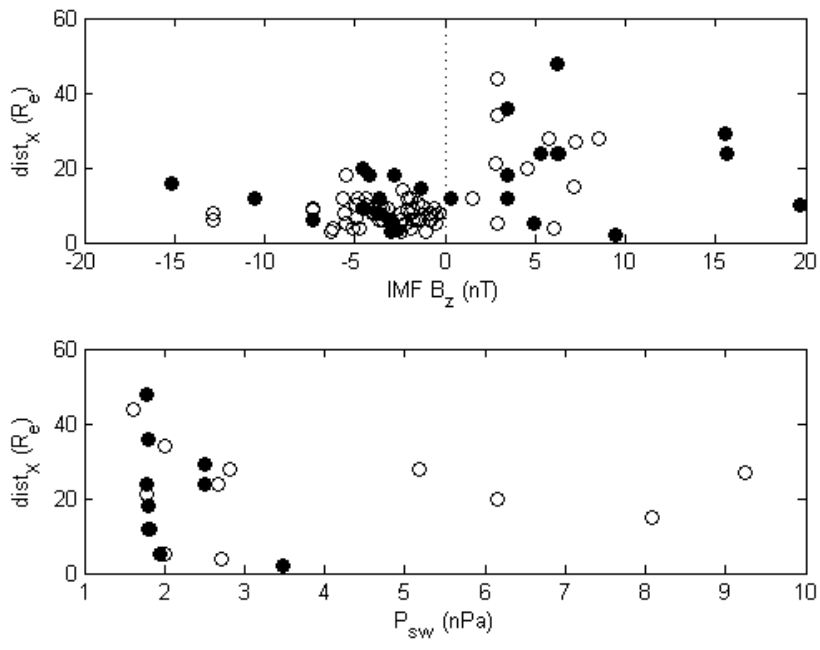

Fig. 8. Distance to reconnection site vs. $B_{z}$ for type- 1 and type2 cusp occurring under steady northward and southward IMF (top) and vs. the solar wind dynamic pressure only for type- 2 cusps occurring also under steady northward IMF (bottom). Empty/full circles correspond to cusp in the Northern/Southern Hemisphere, respectively.

type 2-cusp. The time between the two satellite passes was long enough $(\sim 2 \mathrm{~h})$ for the cusp to fully adjust.

\section{Dispersion and distance to the reconnection site}

\subsection{Method}

The idea in this section is to use the well-known time-offlight effect (Rosenbauer et al., 1975) and lower cut-off energy of the cusp ions (Lockwood and Smith, 1994; Lockwood, 1997) to estimate the distance along the magnetic field lines between the satellite, where the cusp is detected, and the reconnection site. The energy $E_{i}$ of a given zero-pitch angle ion of mass $m$ as a function of the distance to the reconnection site dist $t_{x}$ and the time of flight $t_{i}-t_{0}$, is given by Lockwood (1995, 1997):

$E_{i}=\frac{m}{2}\left(\frac{\text { dist }_{x}}{t_{i}-t_{0}}\right)^{2}$

One may eliminate $m$ and $t_{0}$ by taking two points a two different energies $E_{i}$ or velocities $v_{i}$ (noting that $E_{i}=1 / 2 m v_{i}^{2}$ ). The distance to the reconnection point or line $\left(\right.$ dist $\left._{X}\right)$ is then given by the simple relation:

$\operatorname{dist}_{X}=\frac{\left(t_{2}-t_{1}\right)}{\frac{1}{V_{2}}-\frac{1}{V_{1}}}$

Our method consists in plotting the ion dispersion in a $1 / V$ time spectrogram (where $V$ is the velocity of precipitating ions). The slope of the dispersion gives us directly an estimate of the distance to the reconnection site.
Let us note that this technique generally underestimates the distance to the reconnection site (Lockwood and Smith, 1994). In our case, we have two main limitations. First, we cannot select only zero pitch-angle ions for our calculation but ions having pitch-angles lower than $30^{\circ}$. Also, at middle altitude, the motion of the spacecraft cannot be fully neglected compared to the motion of the open-closed boundary and the convection (Lockwood and Smith, 1994). In fact, there is another technique based on the analysis of the distribution functions of the injected and mirrored ions (Trattner et al., 2004, and references therein) but it is far too heavy to be performed on a large number of samples.

\subsection{Roles of IMF- $B_{z}$ and solar wind pressure}

The calculation of the distance to the reconnection site (dist $t_{x}$ ) is relatively easy as long as the ion dispersion is well defined. We have worked out dist $X$ for the cusp crossings of type 1 and 2 occurring under steady IMF. The results are shown in the top panel of Fig. 8, which displays dist $x$ as a function of $B_{z}$. Empty/full circles are the cusp crossed in the Northern/Southern Hemisphere, respectively. For southward IMF (cusp type 1), reconnection occurs between the two cusps and thus, the distance to the reconnection site is expected to be limited by the size of the dayside magnetosphere. Indeed, it spans between a few $R_{E}$ (when the reconnection site is very close to the exterior cusp) and about $20 R_{E}$ when the reconnection site is near the subsolar point or possibly in the opposite hemisphere. Most of the values found are in fact in the range 8-12 $R_{E}$ (Burch et al., 1982; Menietti and Burch, 1988; Lockwood and Smith, 1994). In contrast, values of dist $_{x}$ for northward IMF should not be limited by the size of the magnetosphere; the reconnection site may be in principle far back in the tail. In practice, the right-hand side of the top panel of Fig. 8 shows nominal values as high as $\sim 30 R_{E}$, with 4 points actually higher than that.

The size of the magnetosphere constitutes a limit for the maximum distance between the reconnection site and a given point in the cusp at a given altitude. Therefore, when the magnetosphere is compressed by a strong solar wind pressure, one should observe smaller values of dist $x$. While magnetic erosion will interfere with the compression of the magnetosphere for southward IMF (both magnetic erosion and solar wind pressure contribute to the size of the dayside magnetosphere), northward IMF cases are more suitable to study the possible effect of the compression of the magnetosphere on the distance to the reconnection site. Bottom panel of Fig. 8 shows dist $x$ as a function of the solar wind dynamic pressure for cusps crossings occurring under northward and stable IMF only. As previously, empty/filled dots represent the cusp detected in the Northern/Southern Hemisphere respectively. Unfortunately, it is difficult to draw any conclusion with the low number of points we have. 


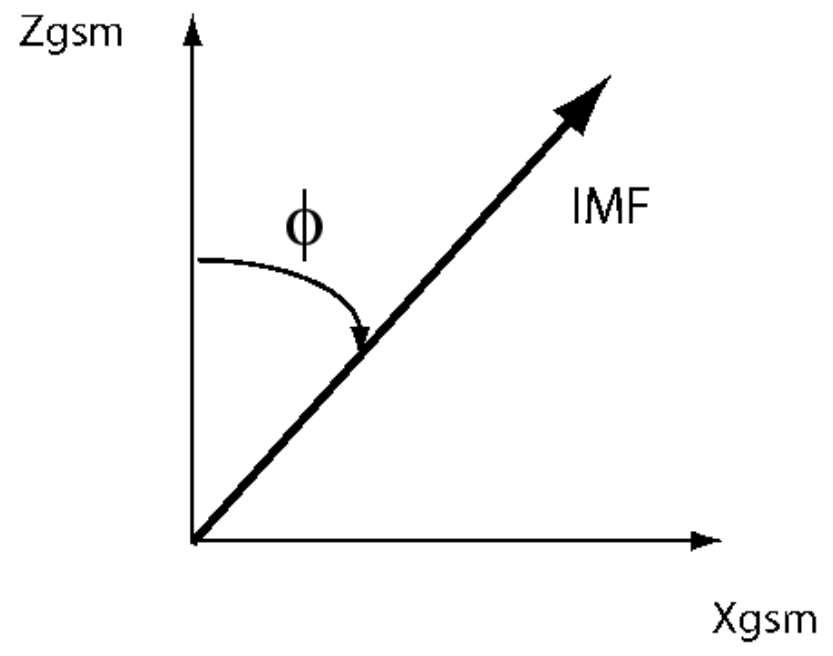

Fig. 9. Definition of the cone angle $(\phi)$.

\subsection{Role of the cone angle of the IMF}

Some hemispheric asymmetries in the solar wind magnetosphere coupling have been reported in the literature (Crooker and Rich, 1993; Østgaard et al., 2005). Although it has been shown that the tilt angle is dominant in determining the hemisphere where reconnection occurs first (Lavraud et al., 2005), we would like to investigate the possible contribution of the cone angle to asymmetries in morphology or in occurrence for instance. Before doing so, let us define what we call the IMF cone angle $\phi$. As the latitudinal location of the reconnection site should ideally (without draping of the IMF lines around the magnetosphere) depend on the sign of $B_{x}$, we have chosen an angle, which changes sign with $B_{x}$ such as depicted in Fig. 9.

We have plotted in Fig. 10 the distance to the reconnection site dist $x$ versus the cone angle $\phi$ of the IMF as defined above. In order to ease the visibility, we have split the type 1 (top) and type 2 cusps (bottom).

Let us start with the top panel of Fig. 10. All points correspond to "normal" time-energy dispersed cusps (decreasing energy with increasing latitude) so it is normal that they are all (but one) observed under IMF whose cone angle are above $90^{\circ}$ in absolute value (i.e. southward IMF). There does not seem to be any tendency in this panel. In fact, we do not expect much asymmetry under southward IMF, the location of the reconnection site being on the dayside magnetopause, between the two cusps.

Cases occurring under northward IMF are shown in the bottom panel of Fig. 10. One can see a clear separation between cusps observed in the Northern Hemisphere (empty circles) when $\phi$ and IMF- $B_{x}$ are positive and cusps observed in the Southern Hemisphere (filled circles) when $\phi$ and $B_{x}$ are negative. It is interesting to note that cusps observed under positive/negative cone angle are predominantly observed
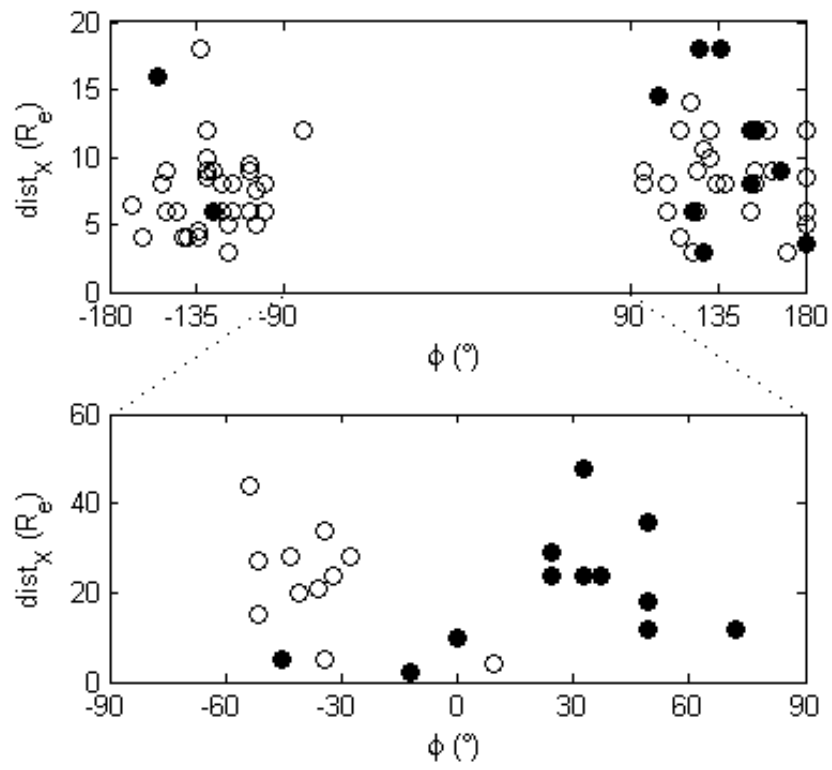

Fig. 10. Distance to the reconnection site vs. cone angle for southward (top) and northward (bottom) IMF. Empty/full circles correspond to cusp in the Northern/Southern Hemisphere, respectively.

in the Southern/Northern Hemisphere, respectively, i.e. precisely in the hemisphere that is supposed to be first encountered by the IMF field lines.

Let us emphasize again that the statistics is quite poor so all this should be investigated with a much larger number of cusp crossings.

\section{Discussions}

\subsection{Discontinuous and irregular cusps}

Differences between discontinuous and irregular cusps under steady IMF have been investigated. It was implicitly postulated at the start of this study that irregular cusp crossings could not possibly result from stable spatial structures. Their highly disturbed and variable nature was off-hand hardly compatible with stable structures under stable IMF. Yet, there exist cases of irregular cusps occurring under stable IMF (14 under southward IMF, and 8 under northward IMF). They are very likely temporal structures due to pulsed reconnection. They might also come from an imprecise calculation of the time required for the IMF to propagate from L1 to Cluster (despite all the cautions).

Yet, interestingly enough, all discontinuous and irregular cusps crossed under stable IMF actually occurred during periods of $B_{y}$-dominated IMF, as reported by several authors (Sandholt et al., 2001; Wing et al., 2001, Pitout et al., 2002). In the framework of anti-parallel reconnection, such conditions are thought to lead to a twisted X-line that encompasses both the high-latitude dayside magnetopause equatorward of 
the cusp and the lobe, poleward of the cusp and that yields a double cusp (injection of magnetosheath particles both equatorward and poleward of the cusp).

\subsection{Anti-parallel vs. component reconnection}

We discuss in this section the possible implications of our results on discontinuous cusps for the recurrent debate regarding anti-parallel versus component reconnection. On the one hand, we have anti-parallel reconnection (Crooker, 1979) which is thought to yield double cusps when the IMF is dominated by its Y-component. Reconnection is then a priori favoured on the flanks of the magnetosphere, relatively far away from magnetic noon (Sandholt et al., 2001). On the other hand, we have component reconnection (Gonzales and Mozer, 1974; Sonnerup, 1974) that basically allows reconnection to occur at both high- and low latitude whatever the IMF direction.

Let us compare these predictions with our observations. We saw in Fig. 6 that both discontinuous and irregular cusps occur systematically under $B_{y}$-dominated IMF, which may give credit to the anti-parallel reconnection hypothesis. But this does not necessarily rule out component reconnection because most cases observed are scattered around magnetic noon and do not occur primarily early in the morning or late in the afternoon, in terms of magnetic local time (bottom of Fig. 6). That is not in favour of anti-parallel reconnection. A priori, only component reconnection could account for this MLT distribution. In addition, we found in paper 1 that component reconnection is very likely to occur instead of anti-parallel reconnection when $B_{y}$ dominates. This was also shown by Trattner et al. (2007).

\subsection{Dispersionless cusp}

In a preliminary version of this study, we had considered a fifth cusp category that we had called "dispersionless cusps". It has to be noted that in their study, Yamauchi and Lundin (1994) had also found what they called "stagnant cusps". Interestingly enough, by increasing the constraints on our selection criteria, all the few dispersionless cusps we had previously identified turned out not to satisfy the criteria and were therefore ruled out or fell into our type 2 category (reverse dispersion).

Furthermore, one can wonder whether a dispersionless ion population correspond to a physical process or is rather an observational feature. Under southward IMF, cusp ions undergo a fast convection. Therefore, the velocity filter effect should occur all the time. In fact, the absence of dispersion in the cusp is very likely an observational feature. For instance, if a satellite crosses the cusp perpendicular to the convection flow, instruments on board will not record any dispersion (Wing et al., 2001). On the other hand, under northward IMF, the convection may be very slow. We have actually observed cusps that exhibit very poor reversed dispersion under these conditions, but there was always a hint of dispersion at the poleward edge of the cusp, near the injection region. Even if a dispersionless component was present equatorward of the dispersed part, we classified those as reverse dispersed. It turns out that the dispersionless parts also contained highenergy ions (above $10 \mathrm{keV} / \mathrm{e}$ ) and were therefore considered as LLBL (closed LLBL in fact), not cusp.

\section{Conclusions}

After having treated the dynamical aspect of the mid-altitude polar cusp (paper 1), we have pursued our statistical study using the same data base. We have analyzed in this paper the large-scale morphology of the cusp with respect to the IMF conditions. Apart from the expected results (dispersed cusps occur under southward IMF and reversed cusp under northward IMF), we have shown that:

- Nicely dispersed cusps represent about half of our observations only.

- Well defined discontinuities in the cusp structure are most of the time due to discontinuities in the solar wind/IMF.

- Yet, there are a number of discontinuous cusps occurring under stable external conditions when the IMF is dominated by its Y-component. Their latitudinal distribution suggests that those cusps very likely result from component reconnection.

Following the rotation of the IMF, we have shown that a discontinuous or irregular cusp, assumably with a dual source of magnetosheath plasma, was often (always in our case) an intermediate step from one type of dispersion to another.

More quantitatively, we have estimated the distance between the satellites and the reconnection site using the slope of the dispersions. Although these estimates are very likely underestimates, they support well the idea that the size of the magnetosphere limits de facto this distance when reconnection between the two cusps occurs under Southward IMF. On the other hand, for Northward IMF, reconnection in the lobe is less constrained and higher distances are logically found.

Acknowledgements. We would like to thank N. Ness at Bartol Research Institute and D. J. McComas at SWRI for providing ACE MAG and SWE data respectively. We would like to thank N. Nagai for providing Geotail data. All ACE and Geotail data were retrieved from CDAWeb. We thank the institutes, which maintain the IMAGE magnetometers array. The IMAGE magnetometer data are collected as a Finnish-German-Norwegian-Polish-Russian-Swedish project. We are grateful to M. Fränz (MPS) for his valuable help and assistance with the Cluster CIS Analysis Tool Interactive (CCATi) and E. Penou (CESR) for his efforts in developing the CL software. FP would like to thank the MPE in Garching for having partly supported this work. This work was supported by the Centre National d'Etudes Spatiales (CNES). It is based on observations with CIS 
embarked on Cluster.

Topical Editor R. Nakamura thanks two anonymous referees for their help in evaluating this paper.

\section{References}

Balogh, A., Carr, C. M., Acuña, M. H., Dunlop, M. W., Beek, T. J., Brown, P., Fornacon, K.-H., Georgescu, E., Glassmeier, K.H., Harris, J., Musmann, G., Oddy, T., and Schwingenschuh, K.: The Cluster Magnetic Field Investigation: overview of in-flight performance and initial results, Ann. Geophys., 19, 1207-1217, 2001, http://www.ann-geophys.net/19/1207/2001/.

Bosqued, J.-M., Sauvaud, J. A., Rème, H., Crasnier, J., Galperin, Yu. I., Kovrazhkin, R. A., and Gladyshev, V. A.: Evidence for ion energy dispersion in the polar cusp related to a northwarddirected IMF, Adv. Space Res., 5, 149-153, doi:10.1016/02731177(85)90130-9, 1985.

Burch, J. L., Reiff, P. H., Heelis, R. A., Winningham, J. D., Hanson, W. B., Gurgiolo, C., Menietti, J. D., Hoffman, R. A., and Barfield, J. N.: Plasma injection and transport in the mid-altitude polar cusp, Geophys. Res. Lett., 9,921-924, 1982.

Burch, J. L., Menietti, J. D., and Barfield, J. N.: DE-1 observations of solar wind-magnetosphere coupling processes in the polar cusp, in: Solar Wind-Magnetosphere Coupling, edited by: Kaminde, Y. and Slavin, J., 441-452, Terra Sceintific., Tokyo, 1986.

Chandler, M. O., Fuselier, S. A., Lockwood, M., Moore, T. E.: Evidence of component merging equatorward of the cusp, J. Geophys. Res., 104, 22623-22634, 1999.

Crooker, N. U.: Dayside merging and cusp geometry, J. Geophys. Res., 83, 951-959, 1979.

Crooker, N. U. and Rich, F. J.: Lobe cell convection as a summer phenomenon, J. Geophys. Res., 98, 13403-13407, 1993.

Escoubet, C. P., Smith, M. F., Fung, S. F., Anderson, P. C., Hoffman, R. A., Baasinska, E. M., and Bosqued, J. M.: Staircase ion signature in the polar cusp: a case study, Geophys. Res. Lett., 19, 1735-1738, 1992.

Escoubet, C. P., Fehringer, M., and Goldstein, M.: The Cluster mission, Ann. Geophys., 19, 1197-1200, 2001,

http://www.ann-geophys.net/19/1197/2001/.

Escoubet, C. P., Berchem, J., Bosqued, J. M., Trattner, K. J., Taylor, M. G. G. T., Pitout, F., Vallat, C., Laakso, H., Masson, A., Dunlop, M., Reme, H., Dandouras, I., and Fazakerley, A.: Two sources of magnetosheath ions observed by Cluster in the mid-altitude cusp, Adv. Space Res., 10, 1528-1536, doi:10.1016/j.asr.2007.04.031, 2007.

Frank, L. A.: Plasmas in the Earth's polar magnetosphere, J. Geophys. Res., 76, 5202-5219, 1971.

Fuselier, S. A., Trattner, K. J., and Petrinec, S. M.: Cusp observations of high- and low-latitude reconnection for northward interplanetary magnetic field, J. Geophys. Res., 105, 253-266, 2000.

Gonzales, W. D. and Moser, F. S.: A quantitative model for the potential resulting from magnetic reconnection with an arbitrary interplanetary magnetic field, J. Geophys. Res., 79, 4186-4194, 1974.

Heikkila, W. J. and Winningham, J. D.: Penetration of Magnetosheath Plasma to Low Altitudes though the Dayside Magnetospheric Cusp, J. Geophys. Res., 76, 883-891, 1971.
Lavraud, B., Thomsen, M. F., Taylor, M. G. G. T., Wang, Y. L., Phan, T. D., Schwartz, S. J., Elphic, R. C., Fazakerley, A., Rème, H., and Balogh, A.: Characteristics of the magnetosheath electron boundary layer under northward interplanetary magnetic field: Implications for high-latitude reconnection, J. Geophys. Res., 110, A06209, doi:10.1029/2004JA010808, 2005.

Lockwood, M. and Smith, M. F.: The variation of reconnection rate at the dayside magnetopause ans cusp ion precipitation, J. Geophys. Res., 97, 14841-14847, 1992.

Lockwood, M. and Smith, M. F.: Low and middle altitude cusp particle signatures for general magnetopause reconnection rate variations: 1. Theory, J. Geophys. Res., 99, 8531-8553, 1994.

Lockwood, M.: Location and Characteristics of the Reconnection X Line Deduced from Low-Altitude Satellite and Ground-Based Observations, 1. Theory, J. Geophys. Res., 100, 21791-21802, 1995.

Lockwood, M.: Energy and pitch-angle dispersions of LLBL/cusp ions seen at middle altitudes: predictions by the open magnetosphere model, Ann. Geophys., 15, 1501-1514, 1997,

http://www.ann-geophys.net/15/1501/1997/.

Lockwood, M., Lanchester, B. S., Frey, H. U., Throp, K., Morley, S. K., Milan, S. E., and Lester, M.: IMF control of cusp proton emission intensity and dayside convection: implications for component and anti-parallel reconnection, Ann. Geophys., 21, 955-982, 2003,

http://www.ann-geophys.net/21/955/2003/.

Němeček, Z., Šimůnek, J., Šafránková, J., and Přech, L.: Spatial and temporal variations of the high-altitude cusp precipitation, Ann. Geophys., 22, 2441-2450, 2004, http://www.ann-geophys.net/22/2441/2004/.

Newell, P. T. and Meng, C.-I.: The cusp and the cleft/LLBL: Lowaltitude identification and statistical local time variation, J. Geophys. Res., 93, 14549-14556, 1988.

Newell, P., Burke, W., Sánchez, E., Meng, C.-I., Greenspan, M., and Clauer, C.: The Low-Latitude Boundary Layer and the Boundary Plasma Sheet at Low Altitude: Prenoon Precipitation Regions and Convection Reversal Boundaries, J. Geophys. Res., 96(A12), 21013-21023, 1991a.

Newell, P., Burke, W., Meng, C.-I., Sanchez, E., and Greenspan, M.: Identification and Observations of the Plasma Mantle at Low Altitude, J. Geophys. Res., 96(A1), 35-45, 1991 b.

Newell, P. T. and Meng, C.-I.: Mapping the dayside ionosphere to the magnetosphere according to particle precipitation characteristics, Geophys. Res. Lett., 19, 609-612, 1992.

Pitout, F., Newell, P. T., and Buchert, S. C.: Simultaneous high- and low-latitude reconnection: ESR and DMSP observations, Ann. Geophys., 20, 1311-1320, 2002,

http://www.ann-geophys.net/20/1311/2002/.

Pitout F., Escoubet, C. P., Bogdanova, Y., Georgescu, E., Fazakerley, A., and Rème, H.: Response of the mid-altitude cusp to rapid rotations of the IMF, Geophys. Res. Lett., 33, L11107, doi:10.1029/2005GL025460, 2006a.

Pitout, F., Escoubet, C. P., Klecker, B., and Rème, H.: Cluster survey of the mid-altitude cusp: 1. size, location, and dynamics, Ann. Geophys., 24, 3011-3026, 2006b, http://www.ann-geophys.net/24/3011/2006/.

Pitout, F., Escoubet, C. P., Klecker, and B., Rème, H.: Cluster survey of the middle altitude cusp - Part 3: Ion density, Ann. Geophys., in review, 2009. 
Reiff, P., Hill, T., and Burch, J.: Solar Wind Plasma Injection at the Dayside Magnetospheric Cusp, J. Geophys. Res., 82(4), 479491, 1977.

Rème, H., Aoustin, C., Bosqued, J. M., Dandouras, I., Lavraud, B., Sauvaud, J. A., Barthe, A., Bouyssou, J., Camus, Th., CoeurJoly, O., Cros, A., Cuvilo, J., Ducay, F., Garbarowitz, Y., Medale, J. L., Penou, E., Perrier, H., Romefort, D., Rouzaud, J., Vallat, C., Alcaydé, D., Jacquey, C., Mazelle, C., d’Uston, C., Möbius, E., Kistler, L. M., Crocker, K., Granoff, M., Mouikis, C., Popecki, M., Vosbury, M., Klecker, B., Hovestadt, D., Kucharek, H., Kuenneth, E., Paschmann, G., Scholer, M., Sckopke, N., Seidenschwang, E., Carlson, C. W., Curtis, D. W., Ingraham, C., Lin, R. P., McFadden, J. P., Parks, G. K., Phan, T., Formisano, V., Amata, E., Bavassano-Cattaneo, M. B., Baldetti, P., Bruno, R., Chionchio, G., Di Lellis, A., Marcucci, M. F., Pallocchia, G., Korth, A., Daly, P. W., Graeve, B., Rosenbauer, H., Vasyliunas, V., McCarthy, M., Wilber, M., Eliasson, L., Lundin, R., Olsen, S., Shelley, E. G., Fuselier, S., Ghielmetti, A. G., Lennartsson, W., Escoubet, C. P., Balsiger, H., Friedel, R., Cao, J.-B., Kovrazhkin, R. A., Papamastorakis, I., Pellat, R., Scudder, J., and Sonnerup, B.: First multispacecraft ion measurements in and near the Earth's magnetosphere with the identical Cluster ion spectrometry (CIS) experiment, Ann. Geophys., 19, 1303-1354, 2001, http://www.ann-geophys.net/19/1303/2001/.

Rosenbauer, H., Gruenwaldt, H., Montgomery, M. D., Paschmann, G., and Skopke, N.: HEOS-2 plasma observations in the distant polar magnetosphere: the plasma mantle, J. Geophys. Res., 80, 2723-2737, 1975.

Russell, C. T., Le, G., and Petrinec, S. M.: Cusp observations of high- and low-latitude reconnection for northward IMF: an alternate view, J. Geophys. Res., 105, 5489-5496, 2000.

Sandholt, P. E., Farrugia, C. J., Moen, J., Noraberg, Ø., Lybekk, B., Sten, T., and Hansen, T.: A classification of dayside auroral forms and activities as a function of IMF orientation, J. Geophys. Res., 103, 23325-23346, 1998a.

Sandholt, P. E., Farrugia, C. J., Moen, J., Cowley, S. W. H., and Lybekk, B.: Dynamics of the aurora and associated convection currents during a cusp bifurcation event, Geophys. Res. Lett., 25, 4313-4316, 1998 b.

Sandholt, P. E., Farrugia, C. J., Cowley, S. W. H., and Lester, M.: Dayside auroral bifurcation sequence during $\mathrm{B}_{y}$-dominated interplanetary magnetic field: Relationship with merging and lobe convection cells, J. Geophys. Res., 106, 15429-15444, 2001.

Smith, M. F. and Lockwood, M.: Earth's magnetospheric cusps, Rev. Geophys., 34, 233-260, 1996.

Sonnerup, B. U. Ö.: The magnetopause reconnection rate, J. Geophys. Res., 79, 1546-1549, 1974.

Trattner, K. J., Fuselier, S. A., Peterson, W. K., Sauvaud, J.-A., Stenuit, H., and Dubouloz, N.: On spatial and temporal structures in the cusp, J. Geophys. Res., 104, 28411-28421, 1999.
Trattner, K. J., Fuselier, S. A., Peterson, W. K., and Carlson, C. W.: Spatial features in the cusp under steady solar wind conditions, J. Geophys. Res., 107(A10), 1288, doi:10.1029/2001JA000262, 2002a.

Trattner, K. J., Fuselier, S. A., Peterson, W. K., Boehm, M., Klumpar, D., Carlson, C. W., and Yeoman, T. K.: Temporal versus spatial interpretation of cusp ion structures observed by two spacecraft, J. Geophys. Res., 107, 1287, doi:10.1029/2001JA000181, 2002b.

Trattner, K. J., Fuselier, S. A., and Petrinec, S. M.: Location of the reconnection line for northward interplanetary magnetic field, J. Geophys. Res., 109, A03219, doi:10.1029/2003JA009975, 2004.

Trattner, K. J., Fuselier, S. A., Yeoman, T. K., Carlson, C., Peterson, W. K., Korth, A., Rème, H., Sauvaud, J. A., and Dubouloz, N.: Spatial and Temporal Cusp Structures Observed by Multiple Spacecraft and Ground Based Observations, Surv. Geophys., 26, 281-305, 2005.

Trattner, K. J., Mulcock, J. S., Petrinec, S. M., and Fuselier, S. A.: Probing the boundary between antiparallel and component reconnection during southward interplanetary magnetic field conditions, J. Geophys. Res., 112, A08210, doi:10.1029/2007JA012270, 2007.

Wing, S., Newell, P. T., and Ruohoniemi, J. M.: Double Cusp: Model Prediction and Observational Verification, J. Geophys. Res., 106, 25571-25594, 2001.

Wing, S., Newell, P. T., and Meng, C. I.: Cusp properties for $\mathrm{B}_{y}$ dominant IMF, Proceedings of the NATO Advances Research Workshop on Multiscale Processes in the Earth's Magnetosphere, NATO science series II. Mathematics, Physics and Chemistry, 178, 149-174, 2004.

Wing, S., Newell, P. T., and Meng, C. I.: Cusp Modeling and Observations at Low Altitude, Surv. Geophys., 26, 341-367, 2005.

Woch, J. and Lundin, R.: Temporal magnetosheath plasma injection observed with Viking - A case study, Ann. Geophys., 9, 133142, 1991.

Yamauchi, M. and Lundin, R.: Classification of large-scale and meso-scale ion dispersion patterns observed by Viking over the cusp-mantle region, in: Physical Signatures of Magnetospheric Boundary Layer Process, edited by: Holtet, J. A. and Egeland, A., pp. 99-109, Kluwer Academic Publishers, Dordrecht, Netherlands, 1994.

Østgaard, N., Mende, S. B., Frey, H. U., and Sigwarth, J. B.: Simultaneous imaging of the reconnection spot in the opposite hemispheres during northward IMF, Geophys. Res. Lett., 32, L21104, doi:10.1029/2005GL024491, 2005. 